\title{
THE STATES OF A BANACH ALGEBRA GENERATE THE DUAL
}

\author{
by ALLAN M. SINCLAIR \\ (Received 8th March 1971)
}

In this paper we prove that the states of a unital Banach algebra generate the dual Banach space as a linear space (Theorem 2). This is a result of R. T. Moore (4, Theorem 1(a)) who uses a decomposition of measures in his proof. In the proof given here the measure theory is replaced by a Hahn-Banach separation argument. We shall let $\boldsymbol{A}$ denote a unital Banach algebra over the complex field, and $D(1)$ denote $\left\{f \in A^{\prime}:\|f\|=f(1)=1\right\}$ where $A^{\prime}$ is the dual of $\boldsymbol{A}$. The motivation of Moore's results is the theorem that in a $C^{*}$-algebra every continuous linear functional is a linear combination of four states (the states are the elements of $D(1))$ (see $(2,2.6 .4,2.1 .9,1.1 .10)$ ).

Recall that the numerical index $n(A)$ of $A$ is defined by

$$
n(A)=\inf \{v(a): a \in A,\|a\|=1\}
$$

where $v(a)=\sup \{|f(a)|: f \in D(1)\}$ [1, Definition 4.9]. We show that the closed balanced convex hull of the states of a unital Banach algebra contains the dual ball of radius the numerical index (Corollary 4). We denote the convex hull of a subset $F$ of a linear space by co $F$, and the closed unit ball

in a Banach space $X$ by $X_{1}$.

$$
\{x \in X:\|x\| \leqq 1\}
$$

I wish to thank R. T. Moore for preprints, and F. F. Bonsall for bringing Moore's work to my notice.

1. Lemma. Let $F$ be a finite set of complex numbers each of modulus 1 , and let $\eta$ be the radius of the largest disc centre the origin that is contained in the convex hull of $F$. Then co $\{\beta D(1): \beta \in F\}$ is a $\sigma\left(A^{\prime}, A\right)$-compact subset of $A^{\prime}$ containing $\eta n(A) A_{1}^{\prime}$.

Proof. Let $F=\left\{\beta_{1}, \ldots, \beta_{n}\right\}$. If each $\beta_{j} D(1)$ has the $\sigma\left(A^{\prime}, A\right)$-topology, and if the product

$$
\beta_{1} D(1) \times \ldots \times \beta_{n} D(1) \times[0,1] \times \ldots \times[0,1]
$$

has the product topology, then the product is compact. The subset $E$ of the product consisting of those elements $\left(\beta_{1} f_{1}, \ldots, \beta_{n} f_{n}, \alpha_{1}, \ldots, \alpha_{n}\right)$ such that $\alpha_{1}+\ldots+\alpha_{n}=1$ is a closed subset of the product. The map $\theta$ from $E$ with the product topology into $\left(A^{\prime}, \sigma\left(A^{\prime}, A\right)\right)$ given by

$$
\theta\left(\beta_{1} f_{1}, \ldots, \beta_{n} f_{n}, \alpha_{1}, \ldots, \alpha_{n}\right)=\alpha_{1} \beta_{1} f_{1}+\ldots+\alpha_{n} \beta_{n} f_{n}
$$


is continuous. The image of $\theta$, which is equal to $\operatorname{co}\{\beta D(1): \beta \in F\}$, is thus $\sigma\left(A^{\prime}, A\right)$-compact.

Let $f$ be in $\eta n(A) A_{1}^{\prime}$, and suppose that $f$ is not in $\operatorname{co}\{\beta D(1): \beta \in F\}$. By a separation form of the Hahn-Banach Theorem (3, Theorem V.2.10, p. 417), there is an $x$ in $A$ (3, Theorem V.3.9, p. 421) and an $\varepsilon>0$ such that $\|x\|=1$ and $\operatorname{Re} f(x)-\varepsilon \geqq \operatorname{Re} g(x)$ for all $g$ in co $\{\beta D(1): \beta \in F\}$. Since co $F$ contains the disc centre the origin with radius $\eta$ in the complex plane,

$\sup \{\operatorname{Re} g(x): g \in \operatorname{co}\{\beta D(1): \beta \in F\}\} \geqq \eta \cdot \sup \{|g(x)|: g \in D(1)\}$.

Therefore

$$
\operatorname{Re} f(x) \geqq \varepsilon+\eta v(x) \geqq \varepsilon+\eta n(A)
$$

which proves that $f$ is not in $\eta n(A) A_{1}^{\prime}$. This gives a contradiction and completes the proof.

Let $H\left(A^{\prime}\right)$ be the real linear subspace of $A^{\prime}$ generated by $D(1)$. The elements of $H\left(A^{\prime}\right)$ are called hermitian functionals [4].

2. Theorem. Let $A$ be a complex unital Banach algebra. Then

$$
A^{\prime}=H\left(A^{\prime}\right)+i H\left(A^{\prime}\right) \text {, }
$$

and $H\left(A^{\prime}\right)$ is a real Banach space under the norm

$$
|f|=\inf \{\alpha+\beta: \alpha \geqq 0, \beta \geqq 0, f=\alpha g-\beta h ; g, h \in D(1)\} .
$$

Proof. An application of Lemma 1 with $F=\{1,-1, i,-i\}$ proves that $A^{\prime}=H\left(A^{\prime}\right)+i H\left(A^{\prime}\right)$. Since $D(1)$ is convex the subset

$$
\{\alpha g-\beta h: \alpha, \beta \in \mathbf{R}, \alpha \geqq 0, \beta \geqq 0 ; g, h \in D(1)\}
$$

of $A^{\prime}$ is a real linear subspace, and so is equal to $H\left(A^{\prime}\right)$. We next prove that if $f$ is in $H\left(A^{\prime}\right)$, then there are $\alpha, \beta \geqq 0$ and $g, h$ in $D(1)$ such that

Let $G$ be the subset of

$$
|f|=\alpha+\beta \text { and } f=\alpha g-\beta h \text {. }
$$

$$
E=D(1) \times D(1) \times[0,|f|+1] \times[0,|f|+1]
$$

of those $(g, h, \alpha, \beta)$ that satisfy $f=\alpha g-\beta h$. Then $G$, which is the intersection of the $\sigma\left(\boldsymbol{A}^{\prime}, \boldsymbol{A}\right)$-closed subsets

$$
\{(g, h, \alpha, \beta):(g, h, \alpha, \beta) \in E, f(x)=\alpha g(x)-\beta h(x)\}
$$

of $E$ as $x$ runs over $A$, is a compact subset of $E$. The function $(g, h, \alpha, \beta) \rightarrow \alpha+\beta$ is continuous on $G$, and therefore the infimum is attained. This proves (1). From (1) and the inequality $\|f\| \leqq|f|$ for $f$ in $H\left(A^{\prime}\right)$ it follows that $\left(H\left(A^{\prime}\right),|\cdot|\right)$ is a normed space.

To prove that $\left(H\left(A^{\prime}\right),|\cdot|\right)$ is complete it is sufficient for us to show that if $f_{0}=0$, and if $f_{n} \in H\left(A^{\prime}\right)$ satisfy $\left|f_{n+1}-f_{n}\right| \leqq 2^{-n}$ for $n=1,2, \ldots$, then there is an $f$ in $H\left(A^{\prime}\right)$ with $\left|f_{n}-f\right|$ tending to zero. Since $\|\cdot\| \leqq|\cdot|$ on $H\left(A^{\prime}\right)$, the series $\Sigma\left(f_{n+1}-f_{n}\right)$ converges in $\boldsymbol{A}^{\prime}$ to an element we denote by $f$. By (1) there are $\alpha_{n}, \beta_{n} \geqq 0$ and $g_{n}, h_{n}$ in $D(1)$ such that

$$
f_{n+1}-f_{n}=\alpha_{n} g_{n}-\beta_{n} h_{n} \text { and }\left|f_{n+1}-f_{n}\right|=\alpha_{n}+\beta_{n} .
$$


For $m=1,2, \ldots$, let

$$
\gamma_{m}=\sum_{n=m}^{\infty} \alpha_{n} \text { and } \zeta_{m}=\sum_{n=m}^{\infty} \beta_{n} .
$$

With convergence in the $\|\cdot\|$-topology we now have

$$
\begin{aligned}
f-f_{m} & =\sum_{n=m}^{\infty}\left(\alpha_{n} g_{n}-\beta_{n} h_{n}\right) \\
& =\gamma_{m} \sum_{n=m}^{\infty} \alpha_{n} \gamma_{m}^{-1} g_{n}-\zeta_{m} \sum_{n=m}^{\infty} \beta_{n} \zeta_{m}^{-1} h_{n} .
\end{aligned}
$$

Further $\sum_{n=m}^{\infty} \alpha_{n} \gamma_{m}^{-1} g_{m}$ and $\sum_{n=m}^{\infty} \beta_{n} \zeta_{m}^{-1} h_{n}$ are in $D(1)$, because $D(1)$ is a $\|\cdot\|$-closed convex subset of $A^{\prime}$. Therefore $f$ is in $H\left(A^{\prime}\right)$, and $\left|f-f_{m}\right| \leqq \gamma_{m}+\zeta_{m}$ for all $m$. This shows that $\left|f-f_{m}\right|$ tends to 0 as $m$ tends to infinity, and completes the proof.

3. Remarks. In proving Theorem 2 we showed that if $f$ is a hermitian functional, then there are $\alpha, \beta \geqq 0$ and $g, h$ in $D(1)$ such that

$$
f=\alpha g-\beta h \text { and }|f|=\alpha+\beta .
$$

If $A$ is a $C^{*}$-algebra, then $\alpha, \beta, g, h$ are uniquely specified by these properties (2, Corollaire 12.3.4, p. 245). Solving the equations $\alpha+\beta=|f|$ and $\alpha-\beta=f(1)$ shows that $\alpha$ and $\beta$ are unique. We now give an example to show that $g$ and $h$ are not unique.

Let $A$ be the complex algebra generated by 1 and $x$ satisfying $x^{3}=0$, and let $A$ have the $\|\cdot\|_{1}$-norm with $\left\{1, x, x^{2}\right\}$ as the basis for $A$. Let $e_{1}, e_{2}, e_{3}$ be the continuous linear functionals on $A$ that are 1 at $1, x, x^{2}$ (respectively) and zero on the other basis elements. In $A^{\prime}$ we have

$$
2 e_{2}+e_{3}=\left(e_{1}+e_{2}+\alpha e_{3}\right)-\left(e_{1}-e_{2}+(\alpha-1) e_{3}\right)
$$

for all $\alpha$ with $0 \leqq \alpha \leqq 1$. Since the norm in $A^{\prime}$ is the $\|\cdot\|_{\infty}$-norm, it follows that $e_{1}+e_{2}+\alpha e_{3}$ and $e_{1}-e_{2}+(\alpha-1) e_{3}$ are in $D(1)$ for $0 \leqq \alpha \leqq 1$. Thus $2 e_{2}+e_{3}$ is in $H\left(A^{\prime}\right)$, and $\left|2 e_{2}+e_{3}\right|=\left\|2 e_{2}+e_{3}\right\|=2$. This gives the required example.

4. Corollary. Let $A$ be a complex unital Banach algebra, and let $B$ be the closed convex hull of $\bigcup\{\beta D(1): \beta \in \mathbf{C},|\beta|=1\}$. Then $n(A) A_{1}^{\prime} \subseteq B \subseteq A_{1}^{\prime}$.

Proof. The corollary will follow if we show that $f$ in $A^{\prime}$ with $\|f\|<n(A)$ implies that $f$ is in $B$. There is a finite set $F$ of complex numbers of modulus 1 whose convex hull contains the disc of radius $\|f\| / n(A)$ centre the origin in the complex plane. By Lemma $1, f$ is in $\operatorname{co}\{\beta D(1): \beta \in F\}$ which is contained in $B$. This completes the proof.

5. Remarks. (a) Corollary 4 is best possible in the sense that $r A_{1}^{\prime} \subseteq B$ implies that $r \leqq n(A)$. We prove this as follows. Let $f$ be in $A^{\prime}$ with $\|f\| \leqq r$, and let $x$ be in $A$. Since $f$ may be approximated by convex sums from

$$
\bigcup\{\beta D(1): \beta \in \mathbf{C},|\beta|=1\},
$$


we obtain $|f(x)| \leqq v(x)$. Thus for each $f$ in $A^{\prime}$ of unit norm and each $x$ in $A$ we have $|f(x)| \leqq v(x) / r$. An application of the Hahn-Banach Theorem implies that $\|x\| \leqq v(x) / r$ for each $x$ in $A$, and therefore $r \geqq n(A)$.

(b) When is $|\cdot|$ on $H\left(A^{\prime}\right)$ equivalent to the dual norm $\|\cdot\|$ of $A^{\prime}$ restricted to $H\left(A^{\prime}\right)$ ? R. T. Moore (letter to the author) has shown that $|\cdot|$ and $\|\cdot\|$ are equivalent on $H\left(A^{\prime}\right)$ if, and only if,

$$
H\left(A^{\prime}\right)=\left\{f \in A^{\prime}: f(h) \in \mathbf{R} \text { for } h \text { hermitian } \in A\right\} .
$$

\section{REFERENCES}

(1) F. F. BONSALL and J. DUNCAN, Numerical ranges of operators on normed spaces and of elements of normed algebras, London Math. Soc. Lecture Note Series, Vol. 2 (1971).

(2) J. Dxмmier, Les $C^{*}$-algèbres et leurs représentations (Gauthier-Villars 1964). 1964).

(3) N. DUNford and J. T. SChwartz, Linear operators, Part 1 (Interscience,

(4) R. T. MOORE, Hermitian functionals on $B$-algebras and characterizations of $C^{*}$-algebras, to appear.

UNIVERSITY OF EDINBURGH, SCOTLAND

UNIVERSITY OF THE WITWATERSRAND, JOHANNESBURG 